\title{
The Grand Challenge in Information Technology and the Illusion of Validity
}

\author{
Michael L. Brodie \\ Verizon Communications Information Technology \\ Boston, MA, USA \\ michael.brodie@verizon.com
}

\begin{abstract}
Rather than progressing in revolutionary, paradigm-shifting steps, Computer Science and Information Technology (IT) follow the much more mundane path called Normal Science by Thomas S. Kuhn in "The Structure of Scientific Revolutions." Scientific and Industrial Revolutions arise only in rare circumstances. The path so often projected by the Industrial IT and Computer Science communities is of revolutions and paradigm shifts. Notwithstanding the Illusion of Validity, the belief in the visions and the claims based on proponent provided justifications, the track record suggests that most claims for emerging technologies are simply false. What should responsible computer scientists and information technologists do when faced with evaluating claims for purported technical advances and new directions? What is a likely outcome of a hot new technology? For example, when will Cooperative Information Systems (CoopISs) come into being? Will we see the claims for Web Services and the Semantic Web realized? Barring rare Nobel Prize-worthy contributions and genuine revolutions the answer is a qualified no - not as claimed and not in the proposed timeframes. Why? Because at their heart is the Grand Challenge of Computer Science: automatically dealing with the "semantics" of data and of computation, e.g., semantic heterogeneity, on which there has been little progress in decades.

This talk looks at the history of claims for emerging technologies, particularly those that contain the Grand Challenge. It reviews some seldom-applied formal and informal methods that can be used to evaluate the potential success of such claims and asks questions such as: What can we expect from Web Services? and How should national funding agencies invest in these areas? The talk concludes after suggesting practical but not novel steps towards making progress, by announcing the next killer app!
\end{abstract}

